\title{
Numerical Study of Enhanced Coercivity of a Magnetically Hard Grain with Thin Surface Layers due to Antiferromagnetic Coupling
}

\author{
H. Fukunaga ${ }^{1}$, Member, IEEE, Y. Yokoi ${ }^{2}$, M. Nakano ${ }^{1}$, T. Yanai ${ }^{1}$, \\ ${ }^{1}$ Graduate School of Engineering, Nagasaki University, Nagasaki 852-8521, Japan \\ ${ }^{2}$ Graduate School of Science and Technology, Nagasaki University, Nagasaki 852-8521, Japan
}

\begin{abstract}
The effects of a thin ferromagnetic layer covering a magnetically hard grain on the coercivity were calculated based on micromagnetic theory. Antiferromagnetic exchange coupling between this thin ferromagnetic surface layer (SF layer) and the hard grain was found to prevent nucleation of reverse domains at the surface of the hard grain and to increase the coercivity. This was effective for hard grains with a layer of reduced magnetic anisotropy on their surfaces whose thickness is comparable with the exchange length. It was also found that a SF layer thickness of several nanometers is suitable. The effects were simulated for a hard grain with SF layers coupled both ferromagnetically and antiferromagnetically. It was found that the coercivity can be enhanced by antiferromagnetically coupled SF layers lying parallel to the easy magnetization axis of the hard grain.
\end{abstract}

Index Terms-Antiferromagnetic coupling, coercive force, exchange coupling, nucleation, permanent magnet.

\section{INTRODUCTION}

$\mathrm{T}$ he effect of grain boundary phases on the coercivity $H_{c}$ of sintered $\mathrm{Nd}-\mathrm{Fe}-\mathrm{B}$ magnets has recently been studied. When nonmagnetic boundaries disrupt the exchange coupling between $\mathrm{Nd}_{2} \mathrm{Fe}_{14} \mathrm{~B}$ grains, magnetization reversal propagates to the whole magnet through only the magnetostatic interaction [1]. This is expected to give a higher $H_{c}$ than that for a magnet with magnetic boundaries $[2,3]$, when $H_{c}$ is determined by the magnetic field required for the propagation of reverse domains. On the other hand, composition analysis by a three-dimensional atom probe suggests that sintered $\mathrm{Nd}-$ $\mathrm{Fe}-\mathrm{B}$ magnets have ferromagnetic boundary phases [4]. In this case, the magnetization reversal will spread to the whole magnet though exchange and magnetostatic interactions. Thus, exchange coupling between the $\mathrm{Nd}_{2} \mathrm{Fe}_{14} \mathrm{~B}$ grain and boundary phases is expected to greatly affect $H_{c}$.

One of important parameters determining the role of boundary phases is the exchange interaction $J$ between boundary and magnetically hard phases. Depending on the on $J$, grain boundaries can function as nucleation sites, a propagating medium for magnetization reversal, and pinning sites for the domain wall. It is also known that grain boundaries coupling antiferromagnetically with a hard grain induce exchange anisotropy and increase $H_{c}$ [5-7]. Some ferromagnetic materials couple antiferromagnetically through the RKKY interaction; $\mathrm{Co}-\mathrm{Ru}-\mathrm{Co}$ has been reported to have the strongest RKKY interaction $\left(-5 \times 10^{-3} \mathrm{~J} / \mathrm{m}^{2}\right)$ [8]. This phenomenon change magnetization process of the coupled substances [9] and is used as an exchange bias for memory materials $[9,10]$. In $\alpha-\mathrm{Fe}(001) / \mathrm{Fe}_{3} \mathrm{O}_{4}(001)$, the two ferromagnetic phases directly exchange couple antiferromagnetically (antiparallelly) and their magnetization process is affected by the exchange interaction [11].

The possibility of antiferromagnetic exchange coupling between $\alpha-\mathrm{Fe}(110)$ and $\mathrm{Nd}_{2} \mathrm{Fe}_{14} \mathrm{~B}(100)$ has recently been predicted by first-principles calculations [12]. The exchange anisotropy induced by the antiferromagnetic coupling is expected to affect $H_{\mathrm{c}}$ of $\mathrm{Nd}_{2} \mathrm{Fe}_{14} \mathrm{~B}$ similarly to the cases in the previous materials [5-10]. We may enhance $H_{\mathrm{c}}$ of magnets by using antiferromagnetic coupling between boundary and hard magnetic phases. However, the magnetic anisotropy of recent hard magnetic materials is much higher than that of the previous materials mentioned above, and it is not clear if we can enhance $H_{\mathrm{c}}$ of magnets with high magnetic anisotropy.

In the present study, we calculated the demagnetization process for a magnetically hard grain with a thin ferromagnetic surface layer (a SF layer) which is antiferromagnetically exchange coupled with the magnetization of the hard grain. It was found that $H_{c}$ can be enhanced by a thin SF layer antiferromagnetically coupled with the hard grain when the exchange coupling is sufficiently strong.

\section{PRINCIPLE OF ENHANCED COERCIVITY BY AN ANTIFERROMAGNETICALLY COUPLED LAYER}

To evaluate the effect of an antiferromagnetically coupled layer on $H_{c}$, we consider the one-dimensional model magnet shown in Fig. 1, which extends infinitely in the $y$ - and $z$-directions. The magnetically hard grain has uniaxial anisotropy, except at the imperfect layer (the IP layer) and the easy magnetization axis lies in the $z$-direction. The IP layer, which is the surface of the hard grain, is thought to lose its magnetic anisotropy due to the presence of crystal imperfections within it. Aharoni $[13,14]$ assumed imperfect crystal anisotropy to explain $H_{c}$ of a magnet. Furthermore, the existence of imperfect crystal anisotropy has been predicted for a $\mathrm{Nd}-\mathrm{Fe}-\mathrm{B}$ magnet [15]. The hard grain is covered by a SF layer, which has a thickness $D_{s}$, and it directly exchange couples antiferromagnetically with the hard grain.

Calculations by Aharoni [13] predict that the IP layer of the hard grain reduces the nucleation field. The solid curve in Fig. 2 shows a typical variation of the nucleation field for a hard grain without a SF layer as a function of the 
logarithm of the IP layer thickness, $D_{i}$. When the hard grain is covered by the SF layer, a field applied in the $z$-direction stabilizes the magnetization configuration shown in Fig. 1 and prevents reverse domain nucleation at the IP layer. Assuming that the magnetization directions in the SF and IP layers, and inside the hard grain are tilted from the $z$ direction by angles of $\pi-\theta_{s}, \theta_{s}$ and $\theta_{p}$, respectively, and neglecting the anisotropy of the SF layer, the magnetic energy $W_{b k}$ stored in the model is given by

$$
\begin{array}{r}
W_{b k}=H_{a}\left\{\mu_{0} M_{i} D_{i}\left(1-\frac{M_{s} D_{s}}{M_{i} D_{i}}\right) \cos \theta_{i}+M_{p} D_{p} \cos \theta_{p}\right\} \\
+J_{p i}\left\{1-\cos \left(\theta_{i}-\theta_{p}\right)\right\}+D_{i} K_{u i} \sin ^{2} \theta_{i},
\end{array}
$$

where $K_{u}$ is the anisotropy constant, $\mu_{0} M$ is the saturation polarization, and $D$ is the layer thickness. The subscripts indicate the layer. Furthermore, $H_{a}$ is the applied field and $J_{p i}$ is the exchange constant between the IP layer and the inside of the hard grain (the perfect (PF) region). As we assume a strong negative exchange interaction between the SF and IP layers and an antiparallel configuration for $M_{\mathrm{s}}$ and $M_{\mathrm{i}}$, the exchange energy, $W E_{\mathrm{AF}}$, stored in the interface between these layers is independent of the magnitude of the applied field. We thus omit $W E_{\mathrm{AF}}$ in (1), which shows that the presence of the SF layer reduces the effective thickness of the IP layer from $D_{i}$ to $D_{i}\left\{1-M_{s} D_{s} /\left(M_{i} D_{i}\right)\right\}$. Therefore, the presence of the SF layer increases the nucleation field, as shown in Fig. 2.

\section{ThreE-Dimensional SimUlation}

\section{1) Calculation Model and Method}

We applied the above concept in three-dimensional calculations. The two models depicted in Fig. 3 were employed in these calculations. The hard grain has an IP layer

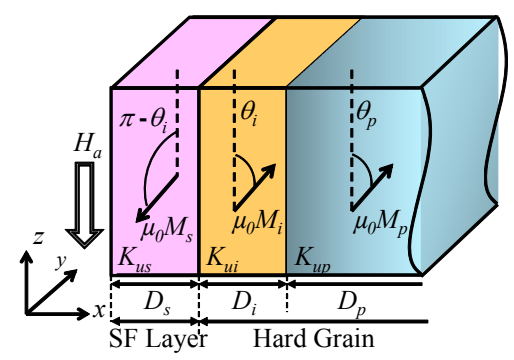

Fig. 1 Model magnet with a thin ferromagnetic layer antiferromagnetically coupled with a magnetically hard grain.

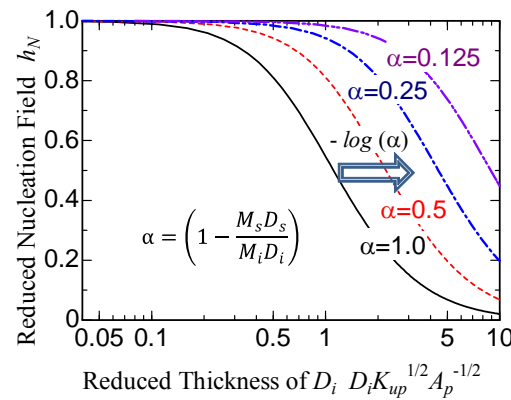

Fig. 2 Effect of an antiparallel coupled layer on the nucleation field. so that it is not magnetically anisotropic and its surfaces are covered by SF layers. The magnetization of the SF layer antiferromagnetically exchange couples with that of the hard grain at all the interfaces in model A (Fig. 3(a)). Since the calculations in Ref. 11 predict that the exchange interaction between $\alpha$-Fe and $\mathrm{Nd}_{2} \mathrm{Fe}_{14} \mathrm{~B}$ will be negative in the $a$-plane but positive in the $c$-plane, we also employed model B (Fig. 3(b)). In model B, the magnetization of the SF layer couples antiferromagnetically with that of the hard grain at interfaces lying parallel to the $x z$ and $y z$ planes, although it couples ferromagnetically at interfaces lying parallel to the $x y$ plane. The assumed positive and negative values of $J_{s-i}$ indicate ferromagnetic and antiferromagnetic couplings, respectively. Both models are composed of 32,768 cubic elements and periodic boundary conditions were applied in the $x$-, $y$-, and $z$ directions. A field was applied in the $z$-direction.

TABLE I lists the simulation parameters used in this investigation. We used $\mathrm{Nd}_{2} \mathrm{Fe}_{14} \mathrm{~B}$ as the hard grain. We set $K_{u}$ of the IP layer to zero as a first-order approximation [13], although various distributions of $K_{u}$ have been employed in previous investigations $[13,14,16,17]$. Employing another distribution may change the optimal SF layer thickness but it will not affect the general tendency of $H_{\mathrm{c}}$. $K_{u}$ was also set to zero for the SF layer to eliminate the effect of the magnetic anisotropy.

In model A, we did not specify the material of the SF layer and we assumed a saturation polarization of $1 \mathrm{~T}$ and an exchange stiffness constant of $2.5 \times 10^{-11} \mathrm{~J} / \mathrm{m}$, both of which are typical values for a ferromagnetic material. We took the SF layer to be $\alpha$-Fe in model B. As mentioned above, negative exchange interactions constants have been determined for $\alpha$ $\mathrm{Fe} / \mathrm{Fe}_{3} \mathrm{O}_{4}$ interface and predicted for $\alpha-\mathrm{Fe} / \mathrm{Nd}_{2} \mathrm{Fe}_{14} \mathrm{~B}$ one; the determined value is $-2.2 \times 10^{-3} \mathrm{~J} / \mathrm{m}^{2}$ for the $\alpha-\mathrm{Fe} / \mathrm{Fe}_{3} \mathrm{O}_{4}$ interface and the maximum predicted value for the $\alpha$ $\mathrm{Fe} / \mathrm{Nd}_{2} \mathrm{Fe}_{14} \mathrm{~B}$ interface is $-1.3 \times 10^{-2} \mathrm{~J} / \mathrm{m}^{2}$. We thus varied the negative exchange constant $J_{\mathrm{s}-\mathrm{i}}$ between $10^{-2}$ and $10^{-4} \mathrm{~J} / \mathrm{m}^{2}$.

The stable direction of the magnetization vector in each element was determined for a given applied field by minimizing the total magnetic energy stored in the model magnet, which includes the anisotropy, exchange, Zeeman, and magnetostatic energies, as given by micromagnetic theory. Demagnetization curves were obtained by varying the magnitude of the applied field. Details of the calculation

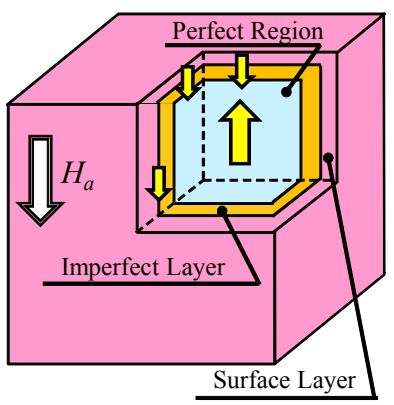

(a) Model A

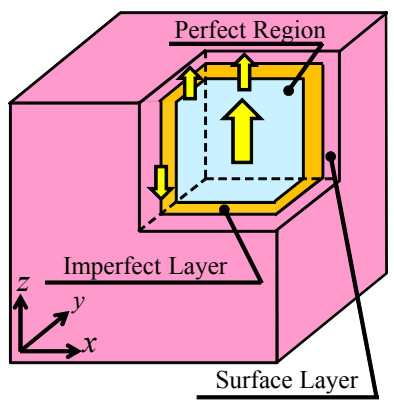

(b) Model B
Fig. 3 Model grains used in three-dimensional simulations. In model A, all the interfaces between the IP and SF layers have negative exchange interactions, whereas those lying parallel to the $x y$ plane have a positive exchange interaction in model $\mathrm{B}$. 
TABLE I SIMULATION PARAMETERS.

\begin{tabular}{|c|c|}
\hline Parameters & Values \\
\hline Model size : $L[\mathrm{~nm}]$ & 48 \\
\hline \multicolumn{2}{|l|}{$\underline{\text { SF layer }}$} \\
\hline Thickness : $D_{s}[\mathrm{~nm}]$ & $0-6$ \\
\hline Exchange stiffness constant : $A[\mathrm{~J} / \mathrm{m}]$ & $2.5 \times 10^{-11}$ \\
\hline \multicolumn{2}{|l|}{ Saturation polarization : $\mu_{0} M[\mathrm{~T}]$} \\
\hline Model A & 1.0 \\
\hline Model B & 2.16 \\
\hline Anisotropy constant : $K_{\mathrm{u}}\left[\mathrm{MJ} / \mathrm{m}^{3}\right]$ & 0 \\
\hline \multicolumn{2}{|l|}{ Hard grain } \\
\hline Exchange stiffness constant : $A[\mathrm{~J} / \mathrm{m}]$ & $8.7 \times 10^{-12}$ \\
\hline Saturation polarization : $\mu_{0} M[\mathrm{~T}]$ & 1.61 \\
\hline \multicolumn{2}{|l|}{ Anisotropy constant : $K_{\mathrm{u}}\left[\mathrm{MJ} / \mathrm{m}^{3}\right]$} \\
\hline Perfect region & 4.5 \\
\hline Imperfect layer & 0 \\
\hline Thickness of : imperfect layer : $D_{\mathrm{i}}[\mathrm{nm}]$ & $0-6$ \\
\hline \multicolumn{2}{|l|}{ Exchange constant between SF layer } \\
\hline Model A & $\begin{array}{l}-1.66 \times 10^{-1}- \\
-1.66 \times 10^{-4}\end{array}$ \\
\hline \multicolumn{2}{|l|}{ Model B } \\
\hline Parallel plane & $\begin{array}{l}-1.3 \times 10^{-1}- \\
-1.3 \times 10^{-4}\end{array}$ \\
\hline Perpendicular plane & $6.5 \times 10^{-3}$ \\
\hline
\end{tabular}

procedure have been reported elsewhere [18].

\section{2) Results and Discussion for Model-A}

Figure 4 shows the calculated demagnetization curves for model A. In the calculation, the SF and IP layer thicknesses were respectively set to 1.5 and $3.0 \mathrm{~nm}$ and the exchange constant between the SF and IP layers, $J_{s-i}$, was varied between $-1.66 \times 10^{-1}$ and $-1.66 \times 10^{-4} \mathrm{~J} / \mathrm{m}^{2}$. When the exchange interaction was weak $\left(J_{S-i}=-1.66 \times 10^{-4}\right)$, the presence of a SF layer gave a lower $H_{c}$ than that (0.226) when no SF layer was present. $H_{c}$ gradually increased with increasing $\left|J_{s-i}\right|$ and was enhanced for $\left|J_{s-i}\right|>1 \times 10^{-3} \mathrm{~J} / \mathrm{m}^{2}$. This value is equivalent to the exchange constant of $\mathrm{Fe}(100) / \mathrm{Fe}_{3} \mathrm{O}_{4}(100)$ [10], but is smaller than that predicted for $\alpha-\mathrm{Fe}(110) / \mathrm{Nd}_{2} \mathrm{Fe}_{14} \mathrm{~B}(100)$ [11]. The enhancement of $H_{c}$ can be attributed to stabilization of the magnetization at the IP layer due to antiferromagnetic coupling between the magnetizations of the SF and IP layers.

Figure 5 shows the effects of $D_{i}$ and $D_{s}$ on the coercivity $h_{c}$, which is the value of $H_{c}$ normalized by the anisotropy field of the PF region of the hard grain. In these calculations, $J_{s-i}$ was set to $-1.66 \times 10^{-2} \mathrm{~J} / \mathrm{m}^{2}$. As shown in Fig. $5, h_{c}$ decreases with increasing $D_{i}$ and increases with increasing $D_{s}$. The observed enhancement of $h_{c}$ saturated at around $D_{s}=4.5 \mathrm{~nm}$, which suggests that a SF layer that is several nanometers thick is sufficient to suppress reverse domain nucleation from the IP layer. For the grain with $D_{i}=3.0 \mathrm{~nm}$, the saturated $h_{c}$ value is approximately two times higher than that for $D_{s}=0$. On the other hand, the effect of the SF layer was not significant for a thick IP layer. This is thought to be because magnetization reversal initiates inside the IP layer, which is far from the interface between the SF and IP layers. Thus, the presence of a SF layer is effective when the thickness of the IP layer is comparable to the exchange length of the hard grain $(1.4 \mathrm{~nm})$.

Indirect exchange coupling between the IP and SF layers via the RKKY interaction [8] seems to have a similar effect as direct interaction. Regardless of direct or indirect exchange coupling, a large $\left|J_{s-i}\right|$ value exceeding $-1 \times 10^{-3} \mathrm{~J} / \mathrm{m}^{2}$ is required to enhance $H_{c}$.

\section{3) Results and Discussion for Model-B}

As the magnetization configuration of model A has not been realized for real magnets, we also calculated $H_{c}$ for hard grains with both antiferromagnetically and ferromagnetically coupled SF layers, based on the prediction for $\alpha-\mathrm{Fe} / \mathrm{Nd}_{2} \mathrm{Fe}_{14} \mathrm{~B}$

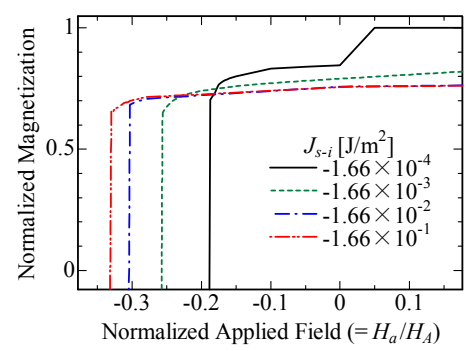

Fig. 4 Effect of magnitude of exchange interaction constant at the interface between SF and IP layers on the demagnetization curves of model A. The SF and IP layer thicknesses were set to 1.5 and $3.0 \mathrm{~nm}$, respectively. The magnetization and applied field are normalized by the saturation magnetization and the anisotropy field of the PF region of the hard grain, respectively.

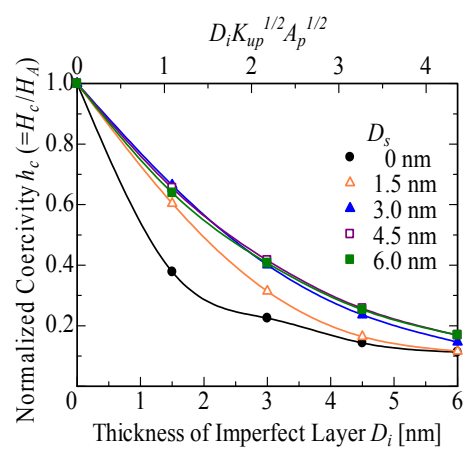

(a) Effect of $D_{i}$

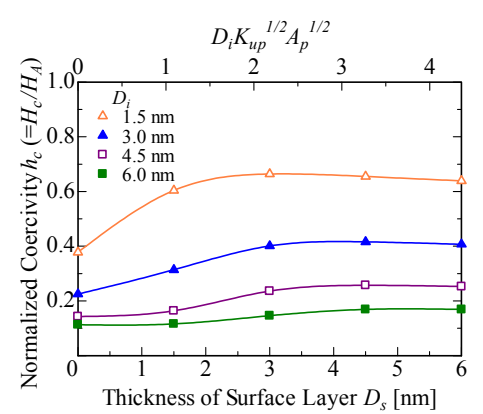

(b) Effect of $D_{s}$

Fig. 5 Effect of thicknesses of (a) IP layer $D_{i}$ and (b) SF layer $D_{s}$ on the normalized coercivity of model A. The upper axis represents the thickness normalized by the exchange length $(=1.4 \mathrm{~nm})$. 


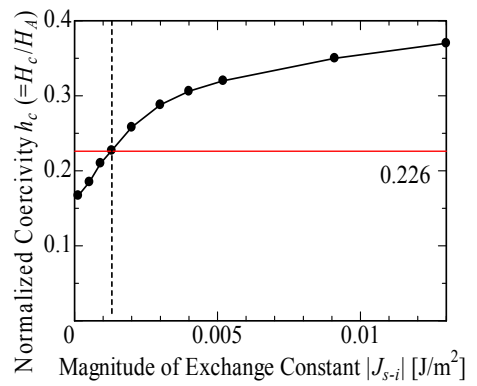

Fig. 6 Effect of magnitude of negative exchange constant between IP and SF layers on the normalized coercivity. The IP and SF layer thicknesses were set to $3 \mathrm{~nm}$.

[12]. Figure 6 shows a plot of $h_{c}$ as a function of the strength of negative exchange interaction. In these calculations, $D_{s}$ and $D_{i}$ were fixed at $3.0 \mathrm{~nm} . h_{c}$ increased with increasing $\left|J_{s-i}\right|$ and it exceeded 0.226 (the $h_{c}$ value for the model without an SF layer) at $\left|J_{a-d}\right|=1.3 \times 10^{-3} \mathrm{~J} / \mathrm{m}^{2}$. This result strongly suggests that the SF layer can enhance $H_{c}$, even if some of the SF layers have ferromagnetic coupling. This behavior can be explained as follows. As our model has IP layers on the hard grain, reverse domain nucleation occurs at some IP layers. IP layers lying parallel to the easy axis of the PF region have a smaller nucleation field $H_{N}$ than IP layers lying perpendicular to the easy axis because nucleation at the perpendicular layers generates a high demagnetization field. In model $\mathrm{B}$, the SF layers lying parallel to the easy axis antiferromagnetically exchange couple with the IP layers with a small $H_{N}$ value, preventing nucleation within them and enhancing $H_{c}$.

Figure 7 shows $h_{\mathrm{c}}$ as a function of $D_{s}$, where $D_{i}$ was set to $3.0 \mathrm{~nm}$. As seen in the figure, $h_{\mathrm{c}}$ exhibits a maximum at about $2.0 \mathrm{~nm}$. This behavior of $h_{\mathrm{c}}$ differs from that shown in Fig. 4 and suggests that strict control of $D_{s}$ is required to enhance $H_{c}$. The presence of a maximum can be attributed to the effect of ferromagnetic coupling, which tends to reduce $H_{\mathrm{c}}$ [19]. For $J_{s-}$ ${ }_{i}=-1.3 \times 10^{-3} \mathrm{~J} / \mathrm{m}^{2}, h_{\mathrm{c}}$ was approximately 1.8 times higher due to the presence of a 2.0 -nm-thick SF layer.

At the present, only $\alpha-\mathrm{Fe}$ is reported to couple antiferromagnetically with $\mathrm{Nd}_{2} \mathrm{Fe}_{14} \mathrm{~B}$, and its role may be clarified experimentally by using thin film process or grain boundary diffusion process.

\section{CONCLUSIONS}

The effects of a thin ferromagnetic layer covering a magnetically hard grain on the coercivity $H_{c}$ were calculated based on micromagnetic theory. A thin surface (SF) layer exchange coupled antiferromagnetically with the hard grain was found to prevent reverse domain nucleation at the surface of the hard grain, and this can enhance $H_{c}$ when the negative exchange interaction is sufficiently strong. The enhancement of $H_{c}$ was remarkable for hard grains with a thin layer that destroys the magnetic anisotropy on their surfaces when its thickness is comparable with the exchange length. A suitable SF layer thickness was found to be several nanometers. We also calculated the effect of SF layers for both ferromagnetic and antiferromagnetic coupling with a hard grain and found that SF layers effectively enhance $H_{c}$ when they are parallel to the easy axis of magnetization of the hard grain.

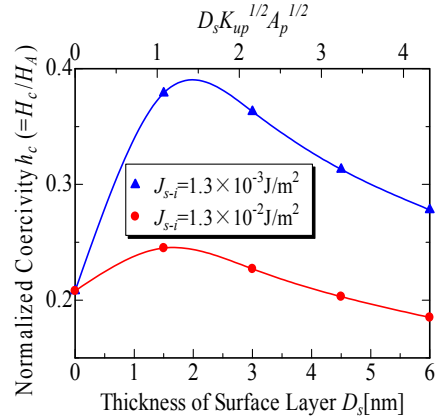

Fig. 7 Effect of SF layer thickness $D_{s}$ on the normalized coercivity $h_{c}$ of model B. $D_{i}$ was set to $3.0 \mathrm{~nm}$.

\section{REFERENCES}

[1] H. Fukunaga, K. Kirini, M. Nakano, T. Yanai, "Effect of Magnetostatic Interaction on Magnetization Reversal Process in Nd-Fe-B Magnets Computer simulation -" J Jpn. Inst. Metals, vol. 76, pp. 43-47, Jan. 2012.

[2] J. Ni, T. Ma, M. Yan, "Changes of microstructure and magnetic properties of Nd-Fe-B sintered magnets by doping Al-Cu," J. Magn. Magn. Mater., vol.323, pp. 2549-2553, Nov. 2011.

[3] R. Ramesh, K. Srikrishna, "Magnetization reversal in nucleation controlled magnets. I. Theory," J. Appl. Phys., vol. 64, pp.6406-6415, Nov. 1988

[4] H Sepehri-Amin, T. Ohkubo, T. Shima, K. Hono "Grain boundary and interface chemistry of an Nd-Fe-B-based sintered magnet," Acta Materialia, vol.60, pp.819-830, Feb. 2012.

[5] W.H. Meiklejhon, "Exchange Anisotropy in the Iron-Iron Oxide System," J. ppl. Phys., vol. 29, pp.454 -455, Mar. 1958.

[6] J.S. Kouvel, C.D. Graham, Jr., and I.S. Jacobs, "Ferromagnetism and antiferromagnetism in disordered Ni-Mn alloys," J. Phys. Radium, Vol. 20, pp.198-202, Feb. 1959.

[7] J.S. Kouvel, C.D. Graham Jr., "Exchange anisotropy in disordered nickel-manganese alloys," J. Phys. Chem. Solids, vol.11, pp.220-225, Oct, 1959.

[8] S.S.P Parkin, "Systematic variation of the strength and oscillation period of indirect magnetic exchange coupling through the $3 \mathrm{~d}, 4 \mathrm{~d}$, and $5 \mathrm{~d}$ transition metals," Phys. Rev. Lett., vol.67, pp. 3598-3601, Dec. 1991.

[9] S.I. Pang, S.N. Piramanayagam, J.P. Wang, "Advanced laminated antiferromagnetically coupled recording media with high thermal stability," Appl. Phys. Lett., vol.80, pp. 616-618, Jan. 2002.

[10] H. Kurt, K. Rode, M.Venkatesan, P Stamenov, J.M.D. Coey, " $\mathrm{Mn}_{3-\mathrm{x}} \mathrm{Ga}$ $(0 \leq \mathrm{x} \leq 1)$ : Multifunctional thin film materials for spintronics and magnetic recording," Physica Status Solidi (B), vol.248, pp. 2338-2344, Oct. 2011.

[11] H. Yanagihara, Y. Toyoda, A. Ohnishi, and E. Kita, "Antiferromagnetic Coupling at the Interface between $\mathrm{Fe}$ and $\mathrm{Fe}_{3} \mathrm{O}_{4}(001)$ Epitaxial Films" Appl. Phys. Express, vol. 1, 111303, Oct. 2008.

[12] Y. Toga, H. Moriya, H. Tsuchiura, A. Sakuma, "First principle study on interfacial electronic structures in exchange-spring magnets" J. Phys. Conf. Ser, vol. 266, 012046, Jan. 2011.

[13] A. Aharoni "Reduction in Coercive Force Caused by a Certain Type of Imperfection," Phys. Rev., vol.119, pp.127-131, Jul. 1960.

[14] C. Abraham, A. Aharoni, "Linear Decrease in the Magnetocrystalline Anisotropy,” Phys. Rev., vol.120, pp.1576-1579, Dec. 1960

[15] G. Hrkac, T.G. Woodcock, C. Freeman, A. Goncharov, J. Dean, T. Schrefl, O. Gutfleisch, "The role of local anisotropy profiles at grain boundaries on the coercivity of $\mathrm{Nd}_{2} \mathrm{Fe}_{14} \mathrm{~B}$ magnets," Appl. Phys. Lett., vol.97, 232511, Dec. 2011.

[16] H. Fukunaga and T. Fukuda, "Effect of magnetic inhomogeneity on magnetization reversal in sintered Nd-Fe-B magnet," Jpn. J. Appl. Phys., vol. 29, pp.1711-1716, Sep. 1990.

[17] A. Sakuma, S. Tanigawa, and M. Tokunaga, "Micromagnetic studies of inhomogeneous nucleation in hard magnets," J. Magn, Magn. Mater, vol. 84, pp.52-58, Mar. 1990.

[18] H. Fukunaga, J. Kuma, Y. Kanai, "Effect of strength of intergrain exchange interaction on magnetic properties of nanocomposite magnets," IEEE Trans. Magn., vol.35, pp.3235-3240, Sep. 1999.

[19] E.F. Kneller, R. Hawing, "The exchange-spring magnet: A new material principle for permanent magnets," IEEE Trans. Magn., vol.29, pp.35883600, Jul. 1991. 